\title{
COMUNICACIÓN/COMMUNICATION
}

Recibido: 05/06/2012 --- Revisado: 30/06/2012 Aceptado: 03/09//2012 --- Publicado: 15/03/2013

\section{LA ADMINISTRACIÓN MUNICIPAL RELACIONAL Y PARTICIPATIVA. CÓMO CONSTRUIR LA IDENTIDAD DE LAS CIUDADES DESDE UNA PERSPECTIVA DE COMUNICACIÓN NEOPÚBLICA}

Concepción Campillo Alhama': Universidad de Alicante. España. concepcion.campillo@ua.es

\section{RESUMEN}

La administración relacional se sustenta en los nuevos roles que pueden desempeñar las asociaciones y colectivos locales en la gestión de lo público. Así, la interacción con el tejido social representa el vínculo a través del cual los administrados pasan a formar parte activa de la organización municipal. Todas estas alianzas forman una compleja red de relaciones institucionales que a su vez se enmarcan, junto con otras estrategias comunicativas, en la política global de comunicación municipal. Los ayuntamientos, como gestores de lo público, deben trabajar en clave relacional para anular dos tendencias igualmente destructivas: la despreocupación municipal por aspectos singulares de la vida ciudadana y la apatía abstencionista de los ciudadanos que impide la implicación colectiva en asuntos de interés general. La gestión estratégica de los vínculos con los colectivos sociales, se identifica con el modelo de gestión neopública y sirve como refuerzo del concepto de ciudadanía a partir de la premisa fundamental de que los ciudadanos constituyen el epicentro de la acción de gobierno; en este contexto, la participación ciudadana se erige como la expresión activa de opiniones de diversa índole sobre temas que afectan o pueden llegar a afectar a los ciudadanos-administrados.

PALABRAS CLAVE: Administración municipal - Ciudadanía - Relaciones sociales Participación - Comunicación.

\footnotetext{
${ }^{1}$ Autor correspondiente:

Concepción Campillo Alhama: Facultad de Ciencias Económicas y Empresariales (Dpto. Comunicación y Psicología Social) Universidad de Alicante. España.

Correo: concepcion.campillo@ua.es
} 


\title{
THE RELATIONAL AND PARTICIPATIVE MUNICIPAL ADMINISTRATION. HOW TO BUILD THE IDENTITY OF THE CITY FROM A COMMUNICATION PERSPECTIVE NEOPÚBLICA
}

\begin{abstract}
Relational management relies on new roles to be played by local associations and groups in the management of public affairs. Thus, the interaction with the social fabric is the link through which the managed actively become part of the municipal organization. These partnerships form a complex network of institutional relationships which in turn are framed, along with other communication strategies, in global politics municipal communication. Municipalities, as managers of the public, must work to override a relational equally destructive two trends: the municipal insouciance unique aspects of city life and abstention apathy of citizens preventing collective involvement in matters of general interest. Strategic management of links with social groups, is identified with the management model and serves as reinforcement neopública the concept of citizenship based on the fundamental premise that citizens are the epicenter of the action of government, in this context, citizen participation stands as the active expression of various kinds of opinions on issues that affect or could affect citizens-administered.
\end{abstract}

KEYWORDS: Municipal Administration - Citizenship - Social Relations - Participation Communication.

\section{INTRODUCCIÓN}

A partir de la instauración de los Ayuntamientos democráticos en España (1979), los entes locales han ido configurando su estructura político-administrativa a partir de distintos modelos de gestión organizacional. Ello permitió cubrir, en los años 80, las necesidades más acuciantes de los municipios, para pasar, a partir de la década de los 90, a solventar otras necesidades complementarias (Maragall, 1997).

Sin embargo, la Administración pública tradicional, inspirada en el modelo organizativo burocrático, con la evolución de los tiempos, ha desarrollado tales disfunciones que ha generado una gran falta de adaptación de las instituciones públicas al actual contexto socioeconómico.

Durante estos treinta años de Ayuntamientos democráticos, las corporaciones locales han estado sumergidas en diferentes etapas históricas que arrancan con el modelo burocrático-taylorista, hasta llegar a la perspectiva contemporánea de la nueva gestión pública (NGP) en la que se desarrollan modelos de gestión postburocráticos ${ }^{2}$ (Ramió,

\footnotetext{
${ }^{2}$ Los modelos de gestión post-burocráticos representan la superación de la dimensión socio-técnica de las organizaciones municipales (centrada en el entorno, los objetivos, las estructuras, los recursos
} 
1999a, 1999b, 1999c, 2002; Olías de Lima, 2001, Hodge, Anthony y Gales, 2003, Arellano y Cabrero, 2005).

En la etapa de la Nueva Gestión Pública (que se enmarca desde 1999 y hasta la actualidad), se manifiestan dos orientaciones teóricas que están relacionadas básicamente con la cultura de las organizaciones públicas (Ramió, 1999a, 1999c; Olías de Lima, 2001) y representan dos formas divergentes de interpretar la gestión político-administrativa: por un lado, nos encontramos con las corrientes neoempresariales, basadas en la economía, la eficacia y eficiencia de los aparatos públicos que proponen una clientelización de los ciudadanos; y por otro, con las corrientes neopúblicas, que depositan un especial énfasis en cuestiones como la repolitización de los cargos públicos, la racionalización y control de la externalización de los servicios, la participación ciudadana y la ética en la gestión.

El peso específico de estas dos orientaciones es bastante desigual (Ramió, 1999a; Olías de Lima, 2001); así, las corrientes neoempresariales representan la mayoría de la literatura organizativa pública y son la fuente de inspiración directa de muchos equipos de gobierno, especialmente en municipios de gran tamaño poblacional. Las neopúblicas, sin embargo, son corrientes minoritarias tanto en el mundo académico como en las propias bases conceptuales sobre las que se desarrollan la innovación y modernización de la Administración pública.

Ambas se erigen, dentro de la teoría organizativa pública, como las tendencias más significativas que sustentan en la actualidad la política de gestión de los Ayuntamientos; sintetizan los numerosos postulados teóricos que se aplican en el ejercicio de la función pública y están muy presentes en el contexto municipal.

\section{ADMINISTRACIÓN NEOEMPRESARIAL VERSUS ADMINISTRACIÓN NEOPÚBLICA}

Dentro de los postulados neoempresariales, destacan diferentes aspectos que nos sitúan ante una visión genérica de lo que supone adoptar en la Administración pública tales planteamientos, frente a las corrientes neopúblicas (Ramió, 1999a, p. 156-158; 1999c).

En primer término, se da la circunstancia de que la orientación neoempresarial se traduce en una acusada fragmentación de la organización administrativa en unidades (más o menos) reducidas que poseen una notable autonomía en cuanto a su gestión económica, patrimonial y laboral; este hecho posibilita que tales unidades se especialicen en la producción de una gama de determinados servicios y se reajusten, con mayor facilidad, a las necesidades de los ciudadanos como clientes o usuarios de tales prestaciones.

financieros, tecnológicos, materiales y humanos, así como los procesos administrativos), para complementarla con una segunda y tercera dimensión basadas en el ámbito político-cultural, el control y la mejora organizativa. 
En este sentido, la creación de organismos que tienen formas jurídicas, alejadas (en mayor o menor grado) del Derecho público (como las empresas públicas y mixtas), es otra de las decisiones habituales de los responsables de aquellas Administraciones, que asumen la orientación neoempresarial como referente explícito del desarrollo de la función pública; adoptando, incluso, la terminología y los conceptos básicos que son utilizados frecuentemente por parte del sector privado. Este impulso de los organismos que tienen formas jurídicas mucho más cercanas al Derecho privado que al público conlleva, por una parte, una cierta atenuación de su responsabilidad con la ciudadanía en cuanto a la ejecución de la gestión, al contrario de lo que sucede cuando tales organismos se encuentran integrados en el propio aparato de la Administración nuclear (neopública); por otra, el consiguiente riesgo de corrupción, derivado de la defensa de intereses particulares que se pueden manifestar en ellos.

Dentro de esta primera orientación, se establece además como segundo postulado la primacía de un Estado que externaliza servicios en la sociedad del bienestar, sobre un Estado intervencionista y del bienestar. Ello supone que las Administraciones públicas pasan de ser proveedoras de servicios públicos a intermediarias que venden servicios; de tal manera que la relación entre la Administración y la ciudadanía queda reducida a una relación entre Administración y ciudadanos que se posicionarían, dentro del marco de la relación administrativa, únicamente como clientes.

Frente a esta primera orientación, e integradas asimismo en el paradigma de la Nueva Gestión Pública, se sitúan las corrientes neopúblicas con una concepción radicalmente opuesta de la gestión de las relaciones administrativas. Desde esta perspectiva, y siguiendo de nuevo a Ramió (1999a, p. 156-158; 1999c), nos encontramos con divergencias sustanciales respecto a los parámetros de las corrientes neoempresariales.

La orientación neopública supone, en primer término, un refuerzo del concepto de ciudadanía a partir de la premisa de la participación ciudadana que permite la expresión activa de las opiniones y posibilita la justificación de la legitimidad política de la Administración pública. Bajo esta perspectiva, se concibe a los administrados como accionistas políticos y económicos de las Administraciones públicas, otorgándoseles muchos más derechos que los asociados, de manera estricta, al rol de cliente. (Ramió, 1999a).

A este primer postulado hay que añadir, el incremento relevante de la cantidad y la calidad de los servicios públicos que supone, por una parte, definir claramente qué ámbitos de la gestión pública son externalizables, garantizando su dirección, control y evaluación por parte de las autoridades públicas desde una perspectiva económica, legal y de defensa de los ciudadanos; por otra, cuáles no pueden serlo desde una óptica basada en las necesidades y el reconocimiento de los derechos propios de la ciudadanía. Este incremento en cuanto a la calidad y cantidad de los servicios conlleva el actuar a partir de principios y valores como la universalidad y la igualdad en la prestación de servicios públicos, en clara contraposición al economicismo que propugnan los modelos neoempresariales. 
El refuerzo de los valores en los empleados públicos así como la creación de una nueva cultura administrativa, asociada tanto a la eficacia y a la eficiencia, como a la ética en la gestión pública, es el tercero de los postulados más significativos de las corrientes neopúblicas.

A partir de estos tres postulados, nos encontramos con dos inconvenientes fundamentales (Ramió, 1999a; Olías de Lima, 2001): en primer término, el hecho de que adoptar como ejes fundamentales de la gestión pública valores como la igualdad, la participación, la cultura pública, la regulación y el rígido control de los servicios públicos externalizados no permita desarrollar una gestión que sea flexible, eficaz y eficiente; en segundo lugar, la circunstancia de tener que soportar, detrás de esta supuesta defensa de los valores públicos, a funcionarios acomodados cuyo objetivo no es otro que la supervivencia de un modelo burocrático que satisfaga sus necesidades particulares frente a las solicitudes, demandas o expectativas manifestadas por la ciudadanía.

Ante tales efectos de carácter negativo que se pueden derivar tanto de la aplicación de postulados neoempresariales como neopúblicos, parece oportuno realizar una reflexión sobre los supuestos básicos de partida que se pueden presentar en el contexto de lo municipal, que atañen a la cultura organizativa y al ejercicio de la función pública.

Según las referencias efectuadas a ambas corrientes, la cultura organizativa en el ámbito municipal (Ramió, 1999c) parte de dos supuestos diferentes: en un primer supuesto, nos situaríamos ante un ámbito de gestión en el que existe una ausencia total o bastante acusada de cultura organizativa, y en el cuál se introducen valores neoempresariales; nos encontramos, en este caso, con una ausencia de enfrentamiento entre orientaciones neopúblicas y empresariales, emergiendo una cultura organizativa de carácter claramente empresarial. En este contexto, los políticos actuarían bajo un rol semejante a los empresarios, los responsables administrativos como directivos o gerentes y el personal de base asumiría el sistema organizativo para obtener, simplemente, un determinado beneficio económico por su rendimiento laboral. Estaríamos ante una organización que se concibe como una empresa, y en la cual se miden los resultados en términos de eficacia y eficiencia, con la ausencia de la perspectiva organizacional neopública que representan valores como la equidad, la igualdad, la participación ciudadana o los impactos sociales de las políticas desarrolladas.

Existe un segundo supuesto que se refiere al hecho de que cuando la cultura neoempresarial se introduce en organizaciones o ámbitos de gestión pública con una sólida cultura organizativa basada en valores públicos, se origina un choque (o al menos cierto enfrentamiento) entre ambas culturas, que puede dar como resultado una cultura organizativa híbrida, resultante de la combinación de valores públicos y empresariales. En esta conexión de valores prevalecerán, mayormente, las orientaciones neopúblicas; no obstante, el impulso que las neoempresariales pueden ejercer sobre la gestión de las organizaciones públicas puede ser sumamente positivo, 
contrarrestando así la defensa de los intereses propios de la organización que van en detrimento de las demandas sociales.

Esta conjunción de orientaciones se traslada a las estructuras organizativas municipales para configurar sistemas compuestos por diferentes unidades 0 subsistemas que se vertebran de manera coordinada en el nivel estratégico, integrando la estructura político-ejecutiva y la administrativa -tecnificada superior y operativa-.

\section{HACIA UNA ADMINISTRACIÓN RELACIONAL Y PARTICIPATIVA}

La administración relacional se basa fundamentalmente en asumir cuáles son los nuevos roles que pueden desempeñar las asociaciones y colectivos locales en la gestión de lo público. Así, la interacción con el tejido social representa el vínculo a través del cual los administrados pasan a formar parte activa de la organización municipal. Para ello los responsables municipales desarrollan estrategias de implicación ciudadana sobre cuestiones que atañen al municipio, y que se materializan mediante reuniones, encuentros formalizados e informales o contactos personales.

Concretamente, en el ámbito municipal, se manifiestan dos aspectos significativos relacionados con las políticas públicas que contribuyen a consolidar una administración próxima a planteamientos neopúblicos: por una parte, la necesidad de superar la perspectiva gerencial-empresarial de los gobiernos locales; por otra, la posibilidad de generar nuevas oportunidades enfocadas a establecer y consolidar alianzas pertinentes con la ciudadanía, sus movimientos asociativos y colectivos diversos (empresarial, cultural, educativo, etc.), la administración central, autonómica y provincial, así como cualquier otro vínculo que responda a los objetivos estratégicos de la organización municipal.

Todas estas alianzas forman una compleja red de relaciones institucionales que a su vez se enmarcan, junto con otras estrategias comunicativas (como la publicidad institucional o la gestión de las relaciones informativas), en la política global de comunicación municipal (Campillo, 2010, 2011). Y en este sentido, los ayuntamientos, como gestores de lo público, deben trabajar en clave relacional para anular dos tendencias igualmente destructivas: la despreocupación municipal por aspectos singulares de la vida ciudadana y la apatía abstencionista de los ciudadanos que impide la implicación colectiva en asuntos de interés general.

Incidimos en la necesidad de diseñar una red de relaciones en la administración municipal, configurada a partir de las interacciones que se pueden generar entre los ayuntamientos y los diversos actores que intervienen (o pueden implicarse) en la consecución de un estado permanente de democracia participativa en el contexto local.

\subsection{Relaciones institucionales y ciudadanía}

La orientación neopública nos proporciona un marco adecuado para el análisis de las 
Administraciones Públicas como propulsoras de diferentes redes interorganizativas, asociativas y ciudadanas que se asientan en los principios básicos de la corresponsabilidad, reciprocidad e integración.

Puig (2003, p. 112) afirmó que se avecinaban tiempos de relaciones públicas, especialmente para las administraciones municipales; y lo justifica en este sentido: "lo propio de la organización municipal es relacionarse/comunicarse públicamente con los ciudadanos para el incremento y sostén de lo común, de lo compartido, de lo público".

A pesar de que el modelo gerencial ${ }^{3}$ se viene imponiendo al modelo clásicoburocrático ${ }^{4}$ en la Administración pública española, no es menos cierto que, aquéllos se desarrollan mayoritariamente desde una orientación estrictamente neoempresarial, obviando las posibilidades que pueden ofrecer estructuras de carácter mucho más participativo (Alguacil, 2005). Así, una y otra orientación condicionan las relaciones que se manifiestan entre los gobiernos locales, las asociaciones y los ciudadanos.

A partir de los planteamientos neopúblicos, los ciudadanos exigen a los responsables políticos que se conviertan en auténticos dinamizadores para propiciar políticas y estrategias concretas, tal y como se refleja en la tabla $n^{0} 1$, que permitan la consecución de cuestiones fundamentales para consolidar esta nueva relación entre administración y administrados.

Tabla no 1. Fundamentos de la administración municipal relacional y participativa

Una relación directa entre la institución municipal, los ciudadanos y sus organizaciones.

Propuestas consensuadas desde argumentos compartidos para la ciudad, como contexto común de todos, y desde la diversidad de las situaciones ciudadanas.

La creación de consejos ciudadanos sectoriales como espacios públicos para la información, el debate,

\footnotetext{
${ }^{3}$ El modelo gerencial parte de la necesidad de dotar de una dirección profesional a la organización municipal. El gerente, como profesional de la gestión organizacional, asume el rol de dirección administrativa sobre la organización del Ayuntamiento y participa, de manera activa, en la planificación y ejecución de políticas y programas locales. Es una figura que surge como resultado de la inadecuación administrativa a nuevas necesidades de las organizaciones públicas y ante la carencia de especialización en la gestión (en la mayoría de las ocasiones) de los políticos. Este modelo se justifica por la necesidad de distinguir el rol directivo-técnico del rol político (asimilado a los cargos electos), separando así el ámbito de la política del de la gestión técnico-administrativa, y reforzando las funciones de tipo político de Alcaldes y Concejales para otorgar responsabilidad directa al gerente en la gestión de la organización municipal.

${ }^{4}$ Los modelos clásico-burocráticos se corresponden con estructuras diseñadas más para desarrollar las funciones tradicionales del control administrativo que para desarrollar políticas prestacionales, propias de organizaciones mucho más operativas. Responden a estructuras administrativas que tienen una concepción estamental muy acusada (dividida en políticos, habilitados nacionales y resto de recursos humanos), además de poseer una orientación marcadamente mecanicista que no facilita, precisamente, una relación fluida con los ciudadanos. Su excesiva longitud vertical conlleva un elevado distanciamiento entre las unidades de dirección estratégica y las unidades meramente operativas, a lo que hay que añadir una excesiva fragmentación sectorial que lleva asociada ciertas disfunciones, vinculadas al elevado índice de divisiones por áreas competenciales, funcionales o sectores administrativos.
} 
el consenso, el pacto, la codecisión, la comunicación, la planificación y la evaluación.

La cogestión de servicios con las asociaciones ciudadanas y pequeñas empresas sociales que permita "ciudadanizar" la gestión municipal.

Una prospectiva de ciudad compartida, con mayor implicación por parte de todos, con alianzas para lo fundamental y servicios que se ajusten a las expectativas creadas.

Un posicionamiento de antijerarquía partidaria, de manera que no se decidan cuestiones que afectan a la ciudadanía porque un partido político ha sido elegido democráticamente en las urnas, sino porque tales cuestiones responden al interés general.

El diálogo múltiple en la gestión de lo público, entendido como una red de relaciones entre los ciudadanos, las asociaciones, los colectivos y los responsables políticos, desde la proximidad y comunicación constante.

Fuente: Puig (2003, p. 111). Elaboración propia.

Todas estas demandas de la ciudadanía adquieren identidad propia dentro de las estrategias globales de comunicación de las administraciones relacionales participativas, ya que implican una constante relación con los ciudadanos, los movimientos asociativos y el tejido social del ámbito de actuación administrativa (Borja, 2003).

$Y$ es precisamente en los municipios, como principal contexto de proximidad ciudadana, donde se manifiesta con mayor incidencia, aún si cabe, la necesidad de atender tales demandas. Para ello, ya no es suficiente aplicar fórmulas tradicionales de comunicación pública (como la publicidad institucional o las relaciones informativas con los medios de comunicación); en el complejo contexto actual de las administraciones es cada vez más habitual la utilización de diversas estrategias de comunicación y relación que permiten generar vínculos sólidos de confianza con el ciudadano: entre ellas, destacamos la interacción personalizada que se manifiesta cuando el ciudadano pone de relieve su condición de administrado en la resolución de trámites o consultas administrativas (de forma presencial u on-line), y las políticas de participación ciudadana, como interacción relacional que se produce con los ciudadanos y representantes de diferentes colectivos sociales a través de la comunicación interpersonal y colectiva, de las redes sociales, plataformas 2.0 y tecnología multimedia (Caldevilla, 2009).

La peculiar idiosincrasia de estas dos vertientes estratégicas exige una aproximación particular para comprender, en toda su extensión, qué representan cada una de ellas en la estrategia de comunicación pública municipal.

A través de las relaciones institucionales se pueden establecer nexos o vínculos entre la ciudadanía, como sistema social, y las organizaciones públicas, como sistemas políticos.

Y, en este sentido, Costa (1992) propone una metodología basada en dos indicadores que permiten identificar a los públicos de una organización: la posición y la 
motivación. Nos parece que tal aportación resulta sumamente relevante ya que nos permite supera el umbral (tradicional) posicional/estructural del mapa relacional, para situarnos en el terreno psicosocial desde dos niveles de análisis interdependientes.

Así, mientras que el mapa taxonómico de los públicos (que representa el número de públicos reales y potenciales) permite visualizar "temáticamente" las diferentes clases de públicos que van a percibir la imagen de la organización, el estudio psicosocial de los vínculos entre los diferentes actores estudia "dinámicamente" las interconexiones que los unen, como complemento operacional del primer mapa. El mapa taxonómico de los públicos, por tanto, es el estadio previo necesario para acceder al análisis relacional.

Figura 1: Variables de la gestión de las relaciones institucionales.

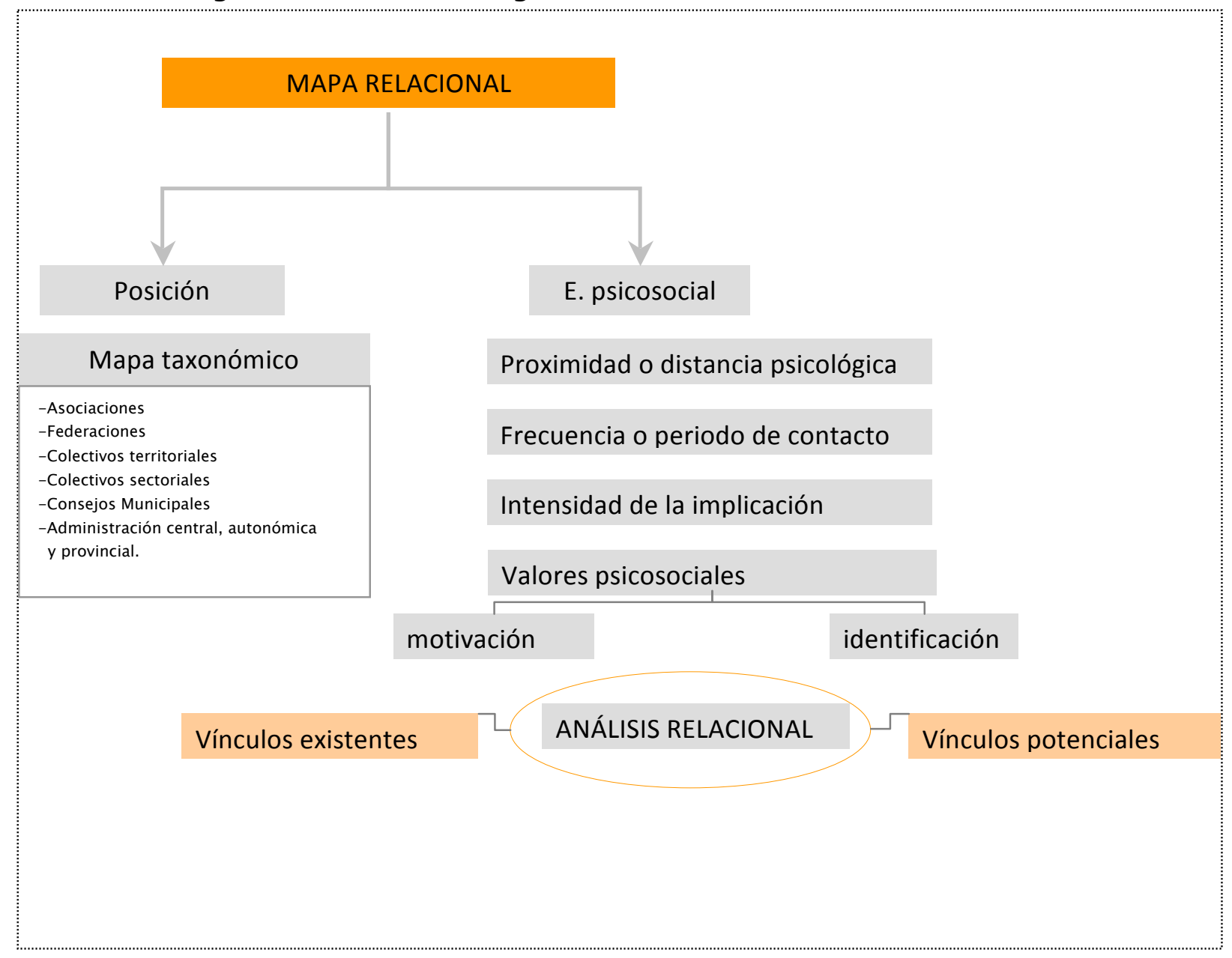

Fuente: Costa (1992) y Xifra $(2003,2010)$. Elaboración propia.

La propuesta de Costa (1992) nos parece especialmente útil en la gestión de las relaciones institucionales de las organizaciones públicas. A partir del mapa de públicos de cada organización se puede establecer cuál es su proximidad o distancia psicológica, la frecuencia o periodo de contacto, la intensidad de la implicación, el carácter y los valores psicosociales (o motivaciones) que caracterizan cada una de 
esas interacciones relacionales, para pasar después a definir y analizar la vinculación de cada tipología de públicos como ejes relacionales de la organización.

El estudio en profundidad de los nexos relacionales es un elemento trascendental para diseñar, con ciertas garantías de éxito, las estrategias de comunicación pública, implementar determinadas actuaciones y controlar permanentemente su desarrollo.

Este sistema de interconexiones, identificado en un momento dado entre la organización pública y su sistema social, no es estático en el tiempo; se completa (y se modifica) a partir de la propuesta de otras interconexiones que convendría introducir a partir de objetivos organizacionales. De esta forma, las organizaciones públicas no sólo ejercitan una vinculación gratificante y duradera con su mapa taxonómico a partir de las interconexiones actualmente establecidas, sino que además planifican estrategias de gestión relacional proactivas orientadas a reforzar su mapa relacional.

La interacción relacional está ligada a unas motivaciones que configuran un cuadro de valores con los que cada público se identifica. Estos vínculos implican, a su vez, que la coincidencia entre el interés recíproco de los sujetos en un proceso comunicativo sea proporcional al grado de intensidad y durabilidad de los vínculos (Xifra, 2003, 2010); en otras palabras: la calidad de la relación impregna cualquier proceso de comunicación pública. Estas interacciones pueden producir efectos positivos o negativos, de grado transcendente, fuerte o débil, y pueden manifestarse como reacciones puntuales o duraderas.

El mapa relacional (o la red de relaciones) de las administraciones municipales se configura a partir de las interrelaciones que se generan entre la organización municipal (también entre sus propios subsistemas) y los diversos actores que intervienen o pueden llegar a intervenir en la consecución de un estado permanente de democracia participativa. Esta red de relaciones se basa en ciertos ejes que, según Alguacil (2005, p. 7), actúan como soportes fundamentales de las relaciones institucionales:

1) Relaciones entre el gobierno local y las entidades sociales: La complementariedad y acoplamiento entre el sector público y las organizaciones sociales es primordial para el desarrollo de estrategias participativas. Las estructuras administrativas aportan recursos económicos y las entidades sociales aportan recursos relacionales. Estas últimas, como mediadoras entre el gobierno local y la base social contribuyen a implementar y legitimar las políticas públicas.

2) Relaciones entre el gobierno local y los ciudadanos: en el desarrollo de la democracia participativa la función pública no se orienta ni a los administrados, ni a los consumidores, ni a los clientes, ni a los usuarios; sino a ciudadanos activos y corresponsables con su municipio. Es imprescindible el trabajo en red, donde cada cual cumple su rol de forma no exclusiva ni excluyente. De esta forma, los grupos informales, los técnicos, los líderes sectoriales o las entidades públicas y privadas actúan como nexos: a través de ellos, y con las estrategias 
adecuadas, los ciudadanos deben incorporarse a experiencias innovadoras de participación.

3) Relaciones inter e intra-administrativas: por una parte, respecto a la coordinación de las distintas administraciones que tienen competencia sobre un territorio, y que representan un aspecto crucial en la gestión pública; por otra, la necesidad de un plan de comunicación relacional interno en las administraciones locales, donde la comunicación y la participación relacional y transversal se asuman desde una posición de desarrollo de competencias, articulando la estructura administrativa para propiciar una verdadera gestión eficiente. En este sentido, las administraciones neopúblicas no asumen la participación como una temática, sector o departamento que deba contemplar cualquier administración, sino que la participación debe ser transversal a las estructuras administrativas, de tal modo que la participación es una parte integrada en todas las partes (o departamentos). Esta cuestión resulta imprescindible para establecer procesos pertinentes de comunicación relacional hacia el exterior.

4) Relaciones de las entidades sociales con su propia base social: la falta de autonomía, de recursos, de formación y de métodos ha hecho de muchas de las asociaciones de base, apéndices de la administración local que compiten por los recursos escasos ofertados por ésta, fundamentalmente para poder mantener sus estructuras asociativas. Su desvinculación de la base social se produce de forma paulatina y ha llevado a muchas asociaciones a verdaderas dinámicas endogámicas, con gran déficit democrático y con serias dificultades para su relevo generacional; en definitiva, con unas estructuras anquilosadas en el pasado, incapaces de asumir los nuevos desafíos de la ciudadanía. La democracia participativa es el contexto que permite una restructuración de las redes sociales que van a actuar como mediadoras frente a una administración que se enfrenta a nuevos fenómenos sociales.

5) Relaciones inter-asociativas: de la misma forma que la administración local precisa de un plan de comunicación relacional interno, el tejido asociativo, en un contexto relacional neopúblico, necesita de su articulación en red para desarrollar la potencialidad sinérgica de un proyecto cooperativo. Esta articulación no es posible si no se dan las condiciones adecuadas para el encuentro, el diálogo y una comunicación bidireccional entre las asociaciones o movimientos, que permita la puesta en común sobre su vocación pública y sobre los principios de la democracia participativa.

En un contexto local sumamente dinámico, en el que intervienen en la configuración de la imagen organizacional diferentes agentes como: los medios de comunicación social, líderes de opinión, movimientos asociativos y tejido social, ciudadanos individuales y plurales, además de otros niveles administrativos, los ayuntamientos tienen que adaptarse de forma permanente a nuevas circunstancias y exigencias sociales, propiciando procesos de comunicación pública que permitan mostrar (y demostrar) a los ciudadanos que constituyen el epicentro de la actuación administrativa. 
Y, en este sentido, la legitimación de la Administración no sólo está relacionada con la ejecución eficaz y eficiente de sus cometidos sino que, además, tal legitimación se justifica por la posibilidad manifiesta de que el propio administrado pueda intervenir en la definición del llamado interés generaF; en este sentido, y siguiendo a Moreu (2005), resulta imprescindible establecer cauces de comunicación pertinentes que fomenten la participación activa de los ciudadanos en el desarrollo de la función pública.

En consecuencia, el establecimiento de un mapa relacional a partir de la taxonomía de públicos y el estudio psicosocial de sus ejes principales representa el paso previo para que cualquier organización municipal pueda establecer las políticas estratégicas relacionales que le van a permitir desarrollar la municipalidad como intercambio, como red de impulsos, de interacciones, de cooperación y colaboración permanente con su sistema social.

Un mapa coherente con los objetivos y las políticas estratégicas de una organización pública facilita, sin duda, la eficacia y la eficiencia administrativa en el desarrollo de las actuaciones. Actúa como eje vertebrador de las políticas de relaciones institucionales y como soporte, para no obviar a determinados colectivos, asociaciones o sectores sociales que pueden amenazar el equilibrio permanente al que deben tender las Administraciones públicas en su gestión relacional.

\subsubsection{La atención al ciudadano desde la nueva gestión pública}

Las Administraciones Públicas del siglo XXI contemplan a los ciudadanos como el verdadero epicentro de la función pública. Su misión se orienta, por tanto, a los servicios solicitados por ellos, a sus demandas como colectivo y a la atención personalizada que requieren.

Por ello, entre los objetivos generales establecidos por las organizaciones públicas se encuentran la mejora de atención a los ciudadanos, así como el establecimiento de sistemas de comunicación recíproca entre la Administración y aquéllos.

Dichos objetivos sólo podrán ser alcanzados:

"a través del desarrollo de un conjunto de estrategias y de la implementación de medidas concretas ligadas a cada una de estas estrategias, para lo cual han de tenerse en cuenta las posibilidades que ofrecen las tecnologías de la información y las comunicaciones" (MAP, 2002, p. 79).

Desde esta primera estrategia relacional administrativa se afrontan cuatro grandes retos, en un modelo de gestión neopúblico (MAP, 2002, p. 81-93); nos referimos a "la

\footnotetext{
5 Término que, siguiendo a Rubio (2005: 324), está relacionado con "el consenso, los valores compartidos y los intereses comunes a largo plazo".
} 
mejora de la calidad y accesibilidad de la información facilitando la comunicación con los ciudadanos, la llamada primera línea de contacto, integrar la demanda de los ciudadanos-usuarios y, por último, verificar los niveles objetivos de calidad".

Cada uno de ellos, comprende aspectos específicos en los que conviene incidir:

a) La mejora de la calidad y accesibilidad de la información: para alcanzar este objetivo, como actuaciones básicas, la Administración puede, entre otras, potenciar los sistemas de almacenamiento informativo en bases de datos e informatizar los procedimientos en su gestión para proporcionar una información de calidad, transparente, fiable, automática, en tiempo real y ajustada a las demandas de los ciudadanos; mejorar la presentación y el lenguaje de los impresos (o formularios) empleados en las relaciones con los ciudadanos para facilitar su comprensión; dar a conocer los servicios y compromisos de la Administración a través de las Cartas de Servicios; generalizar las plataformas de llamadas y fomentar la realización de gestiones administrativas a través del teléfono o del fax; potenciar el uso de la web 2.0 en las relaciones entre la Administración y los ciudadanos a través de redes sociales, plataformas digitales y tecnología multimedia, así como la utilización de puntos de consulta automáticos.

b) La mejora de la primera línea de contacto con el ciudadano: si la primera imagen que percibe el ciudadano en su relación con la Administración resulta determinante a la hora de valorarla, dentro de este objetivo general resulta conveniente desarrollar una estrategia de mejora de atención presencial, de manera que cuando un ciudadano realiza una visita a una Oficina de Atención Ciudadana su percepción sea positiva; y el eje fundamental que está relacionado con la atención presencial es el personal que atiende al ciudadano en sus consultas, quejas, sugerencias, trámites, demandas informativas o de otra índole.

c) Integrar las demandas de los ciudadanos-usuarios: en los modelos de gestión administrativos tradicionales, la oferta de los servicios, su tipología y características venía establecida por la organización pública "ofertante" bajo el criterio de sus técnicos; en los modelos de gestión actuales, sin embargo, la tendencia es que la oferta de servicios sea marcada en función de la demanda de los ciudadanos, ya que son ellos mismos quienes mejor pueden identificar sus necesidades y cómo solventarlas. Esto supone, sin duda, un reto adicional para las Administraciones que tienen que afrontar cambios importantes que suponen una adaptación estructural y operativa continua, ante la naturaleza dinámica de los servicios públicos. En este sentido, es fundamental contar con herramientas que hagan posible conocer la satisfacción de los ciudadanos-usuarios directos del servicio público y sus expectativas sobre él a través de las encuestas de opinión.

d) Verificar los niveles objetivos de calidad: la evaluación de la calidad de la atención al ciudadano ha de realizarse no sólo midiendo el nivel de satisfacción de los ciudadanos, sino también comprobando en qué grado los servicios se ajustan a unos determinados estándares de calidad, previamente fijados por la Administración. Estos patrones se refieren a aspectos externos del servicio y también internos: en cuanto a los primeros, nos referimos, a modo de ejemplo, a la accesibilidad de las instalaciones, identificación y señalización, condiciones ambientales, seguridad o dotación de material. Tales aspectos externos se pueden contemplar a partir de la normativa vigente, por la política de imagen corporativa, por la propia motivación del personal de atención al público o por la demanda de los usuarios del servicio; los aspectos 
internos están relacionados con la garantía y corrección de errores, el nivel de fiabilidad de la información o, incluso, el nivel de quejas o incidencias recibidas.

Estos ambiciosos objetivos conducen a la implementación de un modelo de gestión relacional participativa. Si las organizaciones públicas son capaces de desarrollar estrategias en este sentido, el ciudadano no se identificará como un "simple administrado"; en consecuencia, al sentirse parte de la organización pública, como partícipe de sus políticas estratégicas, establecerá con ella vínculos psicosociales sólidos y estables, basados en la proximidad, la implicación y el reconocimiento de la legitimidad de la organización pública.

\subsubsection{La participación ciudadana como epicentro del sistema administrativo relacional}

El fenómeno del asociacionismo ${ }^{6}$ supone, en primer término, que la consecución efectiva de cualquier régimen democrático conlleva la participación de la ciudadanía en las propias estructuras administrativas donde se localizan los poderes públicos; en segundo lugar, la existencia de una presencia ciudadana suficientemente representativa en la toma de decisiones y el control de las actuaciones administrativas.

Son muchos los municipios españoles ${ }^{7}$, los que ya han elaborado reglamentos de participación ciudadana, o han acometido importantes iniciativas en este sentido como la utilización de encuestas, las audiencias públicas, los encuentros formalizados con colectivos representativos, los foros o mesas sectoriales, los consejos consultivos, los presupuestos participativos, los núcleos de intervención participativa, los foros ciudadanos o la implantación de sistemas electrónicos como cauces de participación (Puig, 2003) y redes sociales (Caldevilla, 2009).

En la Administración municipal, una de las cuestiones prioritarias que se identifica como demanda ciudadana es la apertura de nuevas y diferentes vías que faciliten la implicación de los ciudadanos en la actividad municipal de una manera efectiva y real. Dentro del actual diseño de nuestro modelo participativo, la forma de participación ciudadana por excelencia es la vinculación de los individuos con la esfera pública (y especialmente con el ámbito local) a través de la implicación de éstos en asociaciones o colectivos ciudadanos (Francés, 2006, Pindado, 2008); a través de estas

\footnotetext{
${ }^{6}$ Desde finales de los años 60, y sobre todo, en la década de los 70, surge, en las diferentes democracias occidentales, este fenómeno como mecanismo que trata de influir en la gestión de los asuntos públicos de manera activa.

7 Aquellos que se rigen por la Ley de Grandes Ciudades o Ley 57/2003, de 16 de diciembre, de Medidas para la Modernización del Gobierno Local: esta Ley establece un régimen orgánico específico para los municipios con población superior a los 250.000 habitantes, las capitales de provincia de población superior a 175.000 habitantes, los municipios capitales de provincia, capitales autonómicas o sede de instituciones autonómicas y los municipios cuya población supere los 75.000 habitantes, que presenten circunstancias económicas, sociales, históricas o culturales especiales; constituye un impulso relevante para la modernización de la gestión de los ayuntamientos, promoviendo la participación de los ciudadanos en la gestión de los asuntos locales.
} 
asociaciones se articulan las interrelaciones entre la ciudadanía y las instituciones públicas, responsables de decidir determinadas políticas que pueden afectar a aquellas. Estos colectivos o asociaciones son entidades de muy diversa naturaleza, como clubes deportivos, sindicatos, organizaciones agrarias, organizaciones empresariales, o de carácter socio-cultural (asociaciones de vecinos, culturales, de tercera edad, etc.) que han generado una serie de experiencias participativas dispersas, limitadas y, en muchos casos, poco formalizadas (Borja, 2003).

A partir del barómetro del CIS, de octubre del 2011, y en relación con la asociatividad de la población española, se concluye como dato relevante el hecho de que tan sólo un $12,4 \%$ de la población afirma pertenecer a asociaciones de carácter social o político (las cuales representan la mayor implicación en cuanto a interrelación con los poderes públicos); un índice que puede ser considerado como sumamente discreto. Dentro de este informe, se apunta, además, que amplios segmentos poblacionales afirman incluso no haber pertenecido nunca a un colectivo ciudadano de carácter formal, constatándose así una realidad en torno al fenómeno asociativo (político y social) en nuestro país, que está relacionado con la desafección pública, y que se identifica con una escasa tendencia a involucrarse en el espacio público por parte de la ciudadanía; consecuentemente, y en palabras de Francés $(2006,56)$, "un escenario social diferente, basado en la configuración de un perfil ciudadano no corporativo en busca de nuevas formas de participación y con tendencia a involucrarse en el espacio público" debe ser la respuesta de la Administración a esta situación, para articular mecanismos válidos de innovación democrática local.

Y, en este sentido, dentro de las alternativas de la ciudadanía no asociativa, nos encontramos básicamente con dos cauces: la vía reivindicativa (en cuanto a las metas u objetivos individuales de los ciudadanos) o bien la participación por irrupción (a partir de denuncia o reclamaciones ante la Administración pública).

En estos últimos años ${ }^{8}$, en nuestro país, estamos asistiendo a una cierta reorientación cultural por parte de los diferentes niveles de las administraciones públicas hacia la participación ciudadana como uno de los ejes fundamentales que puede contribuir, notablemente, a mejorar el sistema democrático (Francés, 2006).

A partir del distanciamiento existente entre la clase política y la sociedad civil, es fundamentalmente el sistema político local quien reclama un mayor grado de desarrollo e innovación que contribuya a mejorar la credibilidad y legitimidad de las decisiones públicas. De esta forma, el fenómeno social de la participación ciudadana complementa y refuerza a la democracia representativa (Fernández Ramos, 2005,

\footnotetext{
${ }^{8}$ No es una cuestión novedosa ya que, a partir del establecimiento de la democracia en el año 1978 y el desarrollo de diferentes leyes que atañen a la gestión de la vida municipal, la necesidad de incrementar la colaboración y cooperación ciudadana en la consecución de las políticas públicas y especialmente en la gestión municipal, como contexto inmediato del administrado, es una cuestión prioritaria. Por tanto, nos referimos a un hecho que ya había sido considerado como una de las premisas democráticas básicas durante los años 80 y que recobra fuerza a partir de los parámetros de la nueva gestión pública municipal, asentados a finales de los 90 .
} 
Parés, 2009) para configurar lo que la doctrina jurídica reconoce como democracia participativa.

Esta situación de creciente distancia entre políticos y ciudadanos pone de relieve el desajuste y las disfunciones que se derivan de las estructuras administrativas (y también de los movimientos asociativos) que lleva a sus responsables a orientar la cultura organizacional hacia nuevas estrategias relacionales (de carácter más o menos complejo), según las características intrínsecas de cada ámbito de actuación.

Se plantea, así, una constante búsqueda de nuevas fórmulas que permitan, por una parte, una mayor legitimación de los poderes públicos; por otra, promover procedimientos que incorporen a la masa social a la corresponsabilidad de asumir decisiones que afectan a las estrategias de cambio social.

La participación ciudadana ${ }^{9}$ implica compartir con otros, comunicarse y relacionarse con ellos. A través de ella, no solo se potencia la confianza y la complicidad entre los agentes intervinientes en el proceso sino que el conocimiento sobre determinados asuntos públicos se consolida a partir de esta interacción que va más allá de ser bidireccional; emerge, tal y como apunta Alguacil (2005), con carácter multidireccional, de manera que todos los agentes implicados tienen la posibilidad de asumir, indistintamente, el rol de receptores y emisores de mensajes: ello propicia estados permanentes de aprendizaje, innovación y adaptación mutua.

Es un proceso que se construye a partir de estrategias relacionales para establecer consensos, colaborar y asumir responsabilidades conjuntas de manera continua. En consecuencia, la participación ciudadana no solo representa un medio para alcanzar determinados objetivos sino que, al impulsar constantemente la democracia participativa, representa un fin en sí mismo.

\section{CONCLUSIONES}

\footnotetext{
9 También denominada "participación cooperativa": bajo esta expresión nos referimos a aquella participación en la que la ciudadanía, sin integrarse en la organización pública ni ejercitar funciones públicas, se organiza de forma asociativa para la realización de diversos intereses generales, colaborando con la Administración pública. A partir de la LRBRL/1985 se asume que, en la práctica, la participación ciudadana en los asuntos públicos se articula fundamentalmente a través del tejido asociativo. A través de este mecanismo se incita a los ciudadanos a implicarse en el desarrollo de una verdadera "democracia participativa": por una parte, se encuentran los mecanismos de participación orgánica que derivan en una democracia deliberativa al facilitar, a través de los diferentes órganos participados y participantes, la intervención en la gestión de lo público; son órganos colegiados integrados no sólo por representantes de la Administración municipal, sino parcial o exclusivamente por representantes de determinadas organizaciones sociales. Utilizan diversas denominaciones: Consejos, Comités, Juntas, Comisiones, etc.); en segundo término, la democracia directa que representan los diferentes recursos de participación funcional (desarrollada a través de las iniciativas populares o ciudadanas, las informaciones públicas, las audiencias públicas y las consultas populares; añadiéndose a éstas, aunque a diferente nivel en cuanto a representatividad en la toma de decisiones, las encuestas y estudios de opinión.); y, por último, la democracia asociativa que se materializa a través de la red colectiva que aúna asociaciones, colectivos varios y movimientos reivindicativos o con intereses comunes.
} 
La administración municipal relacional y participativa es la máxima expresión de la gestión neopública de los municipios. En este contexto de proximidad se produce, por una parte, la oferta de los servicios públicos que demanda la ciudadanía como colectivo y la información pertinente junto con la atención personalizada que éstos requieren para acceder a los mismos; por otra, una gestión estratégica de las alianzas o vínculos con los colectivos sociales o grupos de interés, identificados en su mapa taxonómico de públicos, que sirve como refuerzo del concepto de ciudadanía a partir de la premisa fundamental de que los ciudadanos constituyen el epicentro de la acción de gobierno; en este sentido, la participación ciudadana permite la expresión activa de opiniones de diversa índole sobre temas que afectan o pueden llegar a afectar a los ciudadanos-administrados.

Además de la actual crisis económica y financiera en la que nos encontramos inmersos desde el año 2008, Los ayuntamientos se enfrentan a una importante crisis de democracia participativa que se justifica por la desafección y la falta de confianza hacia lo público por parte de la ciudadanía, que aspira a que los responsables políticos de su municipio lideren en los próximos años un proceso de reconstrucción socioeconómica que contribuya a paliar los efectos devastadores de esta crisis.

Para recuperar el protagonismo ciudadano en las decisiones políticas, resulta necesario que, desde los ayuntamientos, se diseñen vías eficientes de participación individual y colectiva a partir de las cuáles se produzcan flujos de información y comunicación de carácter multidireccional que sirvan como reflejo permanente del estado de opinión de la ciudadanía.

Si existe la voluntad real por parte de los ayuntamientos de potenciar un cambio hacia una cultura más participativa, se orientarán los esfuerzos a reforzar el sector asociativo del municipio, a propiciar la participación del ciudadano "no organizado" en la gestión política (mediante foros, encuentros formalizados o juntas participativas sectoriales o de distrito) y a articular mecanismos suficientes de participación colectiva para que los ciudadanos puedan implicarse (de manera activa y pro-activa) en la estrategia de desarrollo socio-económico del territorio.

Esta filosofía de ciudad compartida y gestionada por todos los actores sociales, políticos y económicos que intervienen en su diseño como ente territorial, se identifica con un modelo de gestión neopública que permite a los responsables municipales justificar ante los ciudadanos la legitimidad de su proyecto político como equipo de gobierno y "hacer valer" sus propuestas futuras de cara a los próximos comicios electorales.

\section{REFERENCIAS}

Arellano, D. \& Cabrero, E. (2005). La nueva gestión pública y su teoría de la organización: ¿son argumentos antiliberales? Justicia y equidad en el debate organizacional público. Gestión y Política Pública, XIV (3), 599-618. 
Administración municipal - Ciudadanía - Relaciones sociales - Participación Comunicación

Alguacil, J. (2005). Los desafíos del nuevo poder local: la participación como estrategia relacional en el gobierno local. Polis: revista académica de la Universidad Bolivariana, 12.

Borja, J. (2003). La ciudad conquistada. Madrid: Alianza Ed.

Caldevilla (2009). Democracia 2.0: La política se introduce en las redes sociales. Pensar la Publicidad, III, n² 2, 31-48.

Campillo, C. (2010). Comunicación pública y administración municipal. Una propuesta de modelo estructural. Pensar la Publicidad, IV, no 1, 45-62.

Campillo, C. (2011). Comunicación pública y gestión estratégica municipal. Un estudio exploratorio sobre la agenda temática. "Colección Premios Blas Infante 2010". Sevilla: Instituto Andaluz de Hacienda y Administración Pública.

CIS (2011). Barómetro de octubre, Estudio no 2914. Consultado el 30 de octubre de 2012, Disponible en: http://www.cis.es/cis/opencm/ES/1_encuestas/estudios/ver.jsp?estudio=11904

Costa, J. (1992). Imagen pública: una ingeniería social. Madrid: FUNDESCO.

Fernández Ramos, S. (2005). La información y participación ciudadana en la Administración local. Barcelona: Bosch.

Francés, F.J. (2006). LA PARTICIPACIÓN CIUDADANA DESDE UNA PERSPECTIVA NO ASOCIATIVA Y LOS NUEVOS MECANISMOS DE INNOVACIÓN DEMOCRÁtICA LOCAL: EL CASO PRÁCTICO DE LOS PResupuestos Participativos y los foros CiUdadanos. Tesis doctoral: Universidad de Alicante.

Hodge, Anthony y Gales (2003). Teoría de la organización. Un enfoque estratégico. Madrid: Pearson Prentice Hall (6 $6^{\mathrm{a}}$ Ed.).

Ley 7/1985, de 2 de abril, Reguladora de las Bases del Régimen Local.

MAP- MINISTERIO PARA LAS ADMINISTRACIONES PÚBLICAS (2002) (2a ed.). Libro Blanco para la mejora de los servicios públicos. Una nueva administración al servicio de los ciudadanos. Madrid.

Maragall, P. (1997). Los Ayuntamientos ¿qué eran ? ¿qué son?. Barcelona: Destino.

Moreu, E. (2005). La Administración anunciante. Régimen jurídico de la publicidad institucional. Navarra: Aranzadi.

Olías de Lima, B. (2001) (coord.). La nueva gestión pública. Madrid: Pearson Educación. 
Parés, M. (2009). Tipos de prácticas y experiencias en participación promovidas por la Administración pública: sus límites y radicalidades. En M. Parés (Coord.), Participación y ciudad democrática. Evaluando las nuevas formas de democracia participativa. Barcelona: Ariel.

Pindado, F. (2008). La participación ciudadana en el gobierno local. Madrid: FEMP.

Puig, T. (1994). La ciudad de las asociaciones. Madrid: Ed. Popular.

Puig, T. (2003). La comunicación municipal cómplice con los ciudadanos. Somos una marca de servicios pública con propuestas innovadoras y un estilo entusiasta. Barcelona: Paidós Comunicación.

Ramió, C. (1999a). Teoría de la organización y administración pública. Barcelona: Tecnos/Universitat Pompeu Fabra.

Ramió, C. (1999b). Desarrollo organizativo del Ayuntamiento Gerencial. Barcelona: Universitat Pompeu Fabra.

Ramió, C. (1999c). Corrientes neoempresariales versus corrientes neopúblicas: cultura administrativa, valores públicos y credibilidad social. Un planteamiento radical. Instituciones y Desarrollo, 5. Barcelona: Institut Internacional de la Gobernabilitat de Catalunya.

Ramió, C. (2002). E-Administración i nous models de gestió pública. En I CONGRĖS CATALÀ DE GESTIÓ PÚBLICA. Barcelona.

Rubio, L. (2005). SINGULARIDADES DE LA GESTIÓN DE LA COMUNICACIÓN EXTERNA EN LAS ORGANIZACIONES PÚBLICAS. Tesis Doctoral: Universitat Ramón Lluch.

Sánchez Morón, M. (1992). El sistema político local: fórmulas de gobierno y participación ciudadana. En T. Font i Llovet (Dtor.), Informe sobre el gobierno local. Ministerio de Administraciones Públicas. Madrid-Barcelona: Ministerio para las Administraciones Públicas (MAP), Fundació Carles Pi i Sunyer d'estudis autonòmics i locals.

Xifra, J. (2003). Teoría y estructura de las relaciones públicas. Madrid: Mc Graw Hill.

Xifra, J. (2010). Relaciones públicas y nacionalismo: una aproximación a la construcción nacional desde la perspectiva de las relaciones públicas. Trípodos, 26, 117-132.

\section{Concepción Campillo Alhama}


Profesora Contratada Doctora del Dpto. de Comunicación y Psicología Social de la Universidad de Alicante. Premio BLAS INFANTE 2010, de Estudio e Investigación sobre Administración y Gestión Pública, otorgado por el Instituto Andaluz de Administración Pública (IAAP) a su Tesis Doctoral: Comunicación Pública y Gestión Estratégica Municipal. Licenciada en CC. de la Información (Publicidad y Relaciones Públicas) por la Universidad Complutense de Madrid. Doctora en Sociología por la Universidad de Alicante. Sus líneas de investigación se centran en la comunicación de proximidad de los ayuntamientos, los modelos de comunicación pública, la agenda mediática local y la gestión de eventos creativos. 